\title{
On matching scores for LDA-based face verification
}

\author{
J Kittler, Y P Li and J Matas \\ Centre for Vision, Speech and Signal Processing \\ University of Surrey, Guildford, Surrey GU2 7XH, UK \\ J.Kittler@eim.surrey.ac.uk
}

\begin{abstract}
We address the problem of face verification using linear discriminant analysis and investigate the issue of matching score ${ }^{1}$. We establish the reason behind the success of the normalised correlation. The improved understanding about the role of metric then naturally leads to a novel way of measuring the distance between a probe image and a model. In extensive experimental studies on the publicly available XM2VTS database ${ }^{2}$ using the Lausanne protocol $^{3}$ we show that the proposed metric is consistently superior to both the Euclidean distance and normalised correlation matching scores. The effect of various photometric normalisations ${ }^{4}$ on the matching scores is also investigated.
\end{abstract}

\section{Introduction}

Linear Discriminant Analysis (LDA) is a powerful tool for pattern recognition in general and for face recognition in particular. It was introduced to this application area by Belhumeur in 1996 [1]. As in early independent comparative studies [6] LDA failed to deliver its promise as compared with the Principal Component Analysis method [14] a myriad of alternative techniques have been developed such as the probabilistic model of Moghaddam [10] and the Support Vector Machine method of Jonsson [3]. However, in a recent work these initial set backs have been traced to two major reasons and corrective actions have resulted in LDA's ranking being reinstated. The first problem which contributed to disappointing results is the numerical instability of LDA in face recognition applications due to the huge discrepancy between the observation space dimensionality and the training set size. It has been shown in [5] that this instability can be contained by a very careful selection of the eigenvalue analysis algorithm.

The second contributing factor is the metric used for defining a matching score in the LDA space. With a few exceptions [2], commonly the Euclidean metric has been used in matching $[13,6,7,15,5]$. However, in [4] we have shown that the normalised correlation outperforms the simple Euclidean metric score.

In this paper we revisit the issue of matching score in the LDA space. We establish the reason behind the success of the normalised correlation. The understanding gained about the role of metric then naturally leads to a novel way of measuring the distance between a probe image and a representative of the hypothesised class. In extensive experimental studies on a publicly available database ${ }^{1}$ using a standard protocol $^{2}$ we show 
that the proposed metric is consistently superior to both the Euclidean distance and normalised correlation matching scores. The effect of various photometric normalisations ${ }^{3}$ on the matching scores is also investigated. Although our discussion focuses on the Personal Identity Verification problem where the user claims a certain identity which is then accepted or rejected based on a probe image, we believe that the findings have a much wider significance.

The paper is organised as follows. In Section 2 the Euclidean metric and normalised correlation matching scores are analysed and a novel metric introduced. A description of the experimental setup including the face database used in the study, the experimental protocol, the image registration strategies and photometric normalisation methods is given in Section 3. The experimental results are presented in Section 4. Finally a summary of the main findings and conclusions can be found in Section 5.

\section{Distance Metrics}

It is well known that the Linear Discriminant Analysis (LDA) provides an effective pattern representation. The reason for this is that LDA, in contrast to Principal Components (PCA), focuses on discriminatory content rather than on capturing the variability of the data. Although it is designed to extract first order discriminatory information, in recent experiments in face based personal identity verification LDA has been shown to outperform both linear and nonlinear boundary Support Vector Machines [3]. This may be the consequence of the sparseness of training data in this particular application where only a few gallery images are available in the training set for each client. In such situations only the simplest model, defined in terms of the class mean vector, can be inferred for each client distribution and this is exactly what LDA is able to exploit.

The LDA projection maximises the ratio of between class and within class scatters. In the face recognition or face verification application scenarios the within class covariance matrix is invariably rank deficient, as the number of training images is normally lower than the dimensionality of the image data. For this reason the Linear Discriminant Analysis is performed in a PCA subspace associated with the nonzero eigenvalues of the mixture covariance matrix. In this subspace the LDA axes are known to perform prewhitening of the within class covariances. In other words, the within class covariance matrix becomes an identity matrix. The assumption that each client distribution is Gaussian with mean $\mu_{i}$ and an identity covariance matrix underlies the LDA approach. Under this assumption the optimal metric for face image classification is the Euclidean metric. Accordingly, given a probe image, $\mathbf{x}$, in the LDA space, we can compute a matching score $s$ for the probe and the i-th client mean as the Euclidean distance between the two vectors, i.e.

$$
s_{E}=\sqrt{\left(\mathbf{x}-\mu_{i}\right)^{T}\left(\mathbf{x}-\mu_{i}\right)}
$$

Whereas in the case of PCA representation, a host of different definitions of matching scores have been suggested in the literature, almost all the papers on the use of LDA

\footnotetext{
${ }^{1}$ The work was partically supported by EU Project Banca.

${ }^{2}$ This database is known as extended M2VTS database. For details, see http://www.ee.surrey.ac.uk/Research/VSSP/xm2fdb.

${ }^{3}$ The experimental protocol for the XM2FDB is called the Lausanne protocol. See [9].

${ }^{4}$ The geometric registration is based on the eye positions obtained in three different ways: manually localised eye coordinates, perturbed manually localised eye coordinates and automatically localised eye coordinates [8].
} 
invariably deploy the Euclidean metric. However, we question the merit of this commonly accepted wisdom. There are at least two arguments which raise a doubt about the validity of this particular score measure. First of all the within class covariance matrix used for deriving the LDA projection axes is not rank deficient only thanks to the averaging of the covariance contributions over all the clients. In fact the individual class conditional covariance matrices are rank deficient. As the number of training images for each client is very small, the covariance structure is defined only in a low dimensional subspace of the LDA space. An extrapolation of the structure to the full LDA space is a very dangerous step to make. It would be much safer to define a matching score in the space spanned by the client training images only or ideally just in a one-dimensional space.

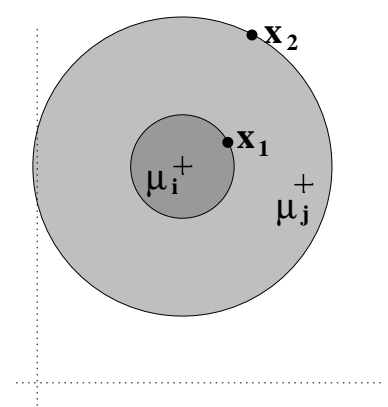

a) Euclidean metric

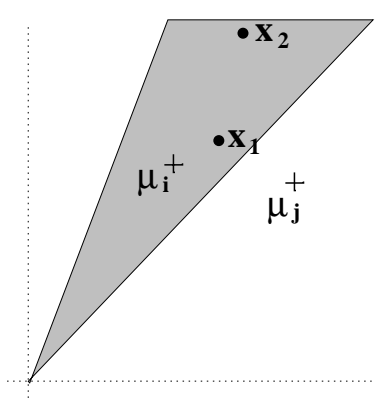

b) Normalised correlation

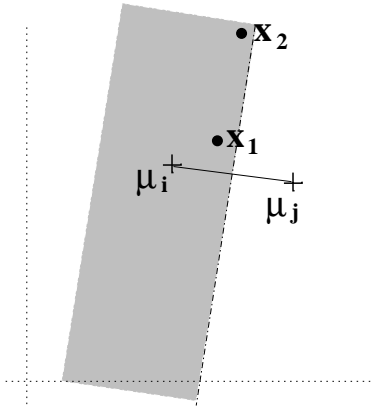

c) Gradient direction metric

Figure 1: Metrics for matching score definition

Second, in the personal identity verification scenario we are not simply interested in the best match but whether the match is good enough. This means that we have to specify a threshold on the score value which defines whether a claimed identity can be accepted, even if the corresponding client mean happens to be the best matching model. The use of the Euclidean distance in this context is marred with difficulties. This can be seen from Figure 1 in which we illustrate the implications of this metric on verifying the claim that the probe image belongs to client $i$. Although actual impostors are not known beforehand, in verification all the other clients may be used to model potential impostors. Suppose that the nearest imposter to client $i$ is client $j$ represented by its mean vector $\mu_{j}$. Let us consider two probe vectors for client $i$ marked in the figure as $\mathbf{x}_{1}$ and $\mathbf{x}_{2}$. Note that both are closer to $\mu_{i}$ than to $\mu_{j}$ but their respective Euclidean distances are very different. Clearly, a threshold on the score for $\mathbf{x}_{2}$ could be much larger than the threshold used with probe $\mathbf{x}_{1}$. Thus a low fixed threshold will in some cases result in unnecessary rejections and its relaxation upward in unwanted false acceptances.

In our earlier work $[5,3]$ we tried to deal with this problem by adopting the normalised correlation as a matching score function. Note that this measure is not a metric. It has been used successfully in the PCA space by [11] but its novel use in conjunction with the LDA representation has been shown to be particularly fruitful. The measure is defined as

$$
s_{N}=\frac{\left\|\mathbf{x}^{T} \mu_{i}\right\|}{\sqrt{\mathbf{x}^{T} \mathbf{x} \mu_{i}^{T} \mu_{i}}}
$$

The normalised correlation projects the probe vector onto the mean vector of the claimed client identity, emanating from the origin. It effectively uses just one dimensional 
space onto which the test data is projected. The magnitude of projection is normalised by the length of the mean and probe vectors. In terms of Figure 1, the normalised correlation tessellates the probe space into hyper cones or hyper frustums with the axes passing through the origin. It is apparent that the normalised correlation score will be insensitive to probe movements in the radial direction defined by the class mean. However, the score will drop in value if the probe moves away from this direction angularly. A threshold on the normalised correlation then defines the acceptance region for each client. In the two class case of Figure 1 the effective acceptance region for client $i$ for a given threshold value is shown by shading. We can see that the measure behaves much better. It reflects the desired variations in sensitivity as a function of probe location.

Notwithstanding the improvements afforded by the normalised correlation, one can still voice some misgivings. The main drawback of the normalised correlation is that the axes of symmetry of the client acceptance cells are constrained to pass the origin. Inspecting Figure 1, this score will not produce the most effective separation of client $i$ from potential imposter $j$. Considering just a pair of classes (with an identity covariance matrix), clearly any distance travelled by the probe image in a direction perpendicular to the line connecting the two mean vectors will not affect the acceptability of the claim. Only a displacement along the line connecting the two means can qualitatively change the decision about a claim. In fact in general it is not the direction defined by the difference of the two means but rather the direction of the gradient of the aposteriori probability function of client $i$. The reason why this direction is decisive is that in high dimensional spaces practically all the data lies on a surface. It will therefore be unlikely to find many observations in the direction parallel to the boundary which would adversely interfere with the decision making process. Our personal identity verification problems falls into this category.

Thus we wish to measure the distance between a probe image $\mathbf{x}$ and the $\mathrm{i}$-th client mean vector $\mu_{i}$ along the direction of the gradient of the i-th class aposteriori probability function $P(i \mid \mathbf{x})$. Using the Bayes formula relating conditional probabilities $P(i \mid \mathbf{x})$ can be computed as

$$
P(i \mid \mathbf{x})=\frac{p(\mathbf{x} \mid i) P(i)}{\sum_{j=1}^{m} p(\mathbf{x} \mid j) P(j)}
$$

where $m$ denotes the number of clients in the database. In (3) $p(\mathbf{x} \mid j)$ is the $\mathbf{j}$-th client measurement distribution and $P(j)$ is the prior probability of the probe image belonging to the $\mathrm{j}$-th client. As mentioned earlier, it is assumed that the client distributions $p(\mathbf{x} \mid j)$ are Gaussian with mean $\mu_{j}$ and an identity covariance matrix, i.e.

$$
p(\mathbf{x} \mid j)=[2 \pi]^{\frac{-d}{2}} \exp \left\{-\frac{1}{2}\left(\mathbf{x}-\mu_{j}\right)^{T}\left(\mathbf{x}-\mu_{j}\right)\right\}
$$

where $d \leq m$ denotes the dimensionality of the LDA space. Differentiating $P(i \mid \mathbf{x})$ in (3) with respect to $\mathbf{x}$ we find the gradient direction $\nabla P(i \mid \mathbf{x})$ as

$$
\nabla P(i \mid \mathbf{x})=\frac{-P(i) p(\mathbf{x} \mid i)}{\left[\sum_{j=1}^{m} p(\mathbf{x} \mid j) P(j)\right]^{2}}\left[\left(\mathbf{x}-\mu_{i}\right) \sum_{j=1}^{m} p(\mathbf{x} \mid j) P(j)-\sum_{j=1}^{m} p(\mathbf{x} \mid j) P(j)\left(\mathbf{x}-\mu_{j}\right)\right]
$$


This can be further simplified as

$$
\nabla P(i \mid \mathbf{x})=\text { const } \times \sum_{j=1}^{m} p(\mathbf{x} \mid j) P(j)\left(\mu_{j}-\mu_{i}\right)
$$

Note that the gradient direction in (6) will be dominated by the worst case impostors, that is by impostors close to the locality of the population of the client of the claimed identity. Moreover, as the length of the gradient vector will have to be normalised anyway the constant on the rhs of (6) can be ignored. Interestingly, any differences in the respective prior probabilities $P(i)$ and $P(j)$ of the true client and impostors claiming access will not affect the direction of the gradient as the i-th term of the sum will be zero. In this respect the priors can also be ignored in the computation of the gradient direction, giving finally a simple formula

$$
\nabla P(i \mid \mathbf{x})=\sum_{\substack{j=1 \\ j \neq i}}^{m} p(\mathbf{x} \mid j)\left(\mu_{j}-\mu_{i}\right)
$$

Now projecting the difference of the probe image and the client mean vector on the gradient direction (7) will give the proposed optimal matching score

$$
s_{O}=\frac{\left\|\left(\mathbf{x}-\mu_{i}\right)^{T} \nabla P(i \mid \mathbf{x})\right\|}{\|\nabla P(i \mid \mathbf{x})\|}
$$

In the next section the above three scores, $s_{E}, s_{N}$ and $s_{O}$ will be compared experimentally on the XM2VTS database.

\section{Experimental Setup}

The proposed metric and the associated matching score is experimentally evaluated on a large publicly available face image database known as the XM2VTS database. XM2VTS is a multimodal database recorder specifically for assessing the performance of multimodal biometric approaches to personal identity verification. In our experiments one of the standard image subsets have been used for which a standard performance characterisation protocol has been defined so that the results of competing algorithms are directly comparable.

XM2VTS database contains 295 subjects. The subjects were recorded in four separate sessions uniformly distributed over a period of 5 months, and within each session two shots were taken each including frontal-view and rotation sequences. In the frontal-view sequence the subjects read a specific text (providing synchronised image and speech data), and in the rotation sequence the head is moved vertically and horizontally in order to capture different views of the anterior part of the head and to provide information useful for 3D surface modelling of the head. Details of this database can be found in [9] or http://www.ee.surrey.ac.uk/Research/VSSP/xm2fdb.html.

The experimental protocol known as the Lausanne evaluation protocol divides the database into 200 clients and 95 impostors. For each client/imposter one frame per shot per session is selected from images of frontal or near frontal views, thus giving 8 images per subject in total. These sets are further divided into training, evaluation and test sets to 
create two distinct configurations of the data. In our experiments we used only Configuration I which is known to be more demanding. The set of impostors is split into two subsets so that the data used for testing is completely independent from that used for finding the LDA axes and the matching score thresholds. The performance is measured in terms of false rejection and false acceptance rates. Details of the this protocol can be found at http://www.idiap.ch/ m2vts/Experiments/xm2vtsdb_protocol_october.ps

The LDA face verification method can work successfully only when the images are properly registered. In our work face registration is performed by an eye position dependent utility. This utility takes four parameters computed from the eye coordinates (rotation, scaling and translation in the horisontal and vertical directions) to crop the face part from the original image and scale it to any desired resolution. Experiments were conducted for different registration methods which depend on the manner the eye coordinates are extracted.

Three different sets of eye coordinates were used for face registration in order to investigate how the registration strategies influence the performance:

- Manual Registration which provides the ground truth eye positions with less than \pm 1 pixel error. The verification errors obtained on manually registered images define a baseline performance.

- Pseudo Registration which is based on eye coordinates generated synthetically by perturbing the manually localised eye positions. The horizontal and vertical coordinates of the eye positions were independently perturbed by adding random displacements drawn from a normal distribution with a zero mean and standard deviation equal to 2 . The objective of using pseudo registration was to investigate how sensitive the verification method was to missregistration.

- Automatic Registration which was obtained by a fully automatic approach based on robust correlation. As for the Pseudo Registration, only the evaluation set and test sets were registered automatically.

Once an image is registered a Photometric Normalisation is applied to compensate for changing illumination. We have experimented with the following methods:

-Zero Mean (ZM): The mean of the image is removed from each pixel value.

-Zero Mean and Unit Variance (ZMST): The mean of the image is removed and the pixel values scaled by their standard deviation.

-Histogram Equalisation (HEQ): The image is histogram equalised.

-Best Fitting Plane (FP): The best fitting plane is subtracted from the image.

-Best Fitting Plane and Unit Variance (FPST): After subtracting the best fitting plane, the pixel values are scaled by their standard deviation.

-Best Fitting Plane and Histogram Equalisation (FPHQ): After subtracting the best fitting plane,the image is histogram equalised.

\section{Experimental results and discussion}

The primary aim of our experiments was to compare the performance of the three different score measures defined in the LDA space in the context of face verification. However, a secondary objective was to investigate the effect of various methods of photometric 
normalisation of the face images on the ranking of these measures. Our strategy was to establish the relative merits of the different photometric normalisation methods on the manually registered images and select only a subset of the most promising approaches for an experimental evaluation of the scores on pseudo registered and automatically registered data.

As mentioned earlier, the experiments were carried out according to the Lausanne protocol which specifies that 200 clients be used for training. For this number of clients the maximum dimensionality of the LDA space is 199. In our earlier work we investigated the relationship between the dimensionality of the LDA space and the verification performance and showed that as the dimensionality of the feature space reduces, the performance monotonically worsens. Although initially the degradation is very gradual, allowing a significant reduction in the computational complexity without a serious performance loss, we opted to experiment in the full LDA space.

Similarly, earlier experimental studies demonstrated that it is beneficial to low pass filter the image data and subsample it. In fact the optimal performance was achieved with a relatively low resolution face images, namely $61 \times 57$ pixels. The results reported in this section have been obtained with this image size.

The results obtained on the manually registered XM2VTS database are shown in Table 1 . The table is divided vertically into three sections presenting the performance figures for the three different score measures. The entries are parameterised by the type of photometric normalisation deployed. Each row reports the results on the evaluation set and test set respectively. The evaluation set, which is independent of the training set, is used to compute the receiver operating characteristics (ROC curve) by varying the client specific threshold and measuring the corresponding false rejection (FR) and false acceptance (FR) rates. The point on the ROC curve for which these two error rates are equal is selected as the operating threshold. The equal error rates and their unweighted sum - the total error (TE) - are noted in the table. The performance of the verification system at this operating threshold is then verified on an independent test set and the results recorded in the last three columns of the table. Note that in general there is a close agreement between the results obtained on the evaluation and test sets which shows that the selected thresholds generalise well.

From the table we can see that the photometric normalisation which involves subtracting the best fitting plane (and the variations on the theme) does not generally improve the system performance. By checking a number of images we noticed that the parameters of the best fitting plane are often dominated by regions of total reflection in the image, rather than by the underlying gradient in illumination. We also observed that removing the image mean did not help much either. This can be explained by noting that the subtraction of the global mean from each image performed a similar role which made the removal of the image mean a superfluous operation. For this reason we limited our subsequent experiments to consistently superior photometric normalisation methods, namely ZMST and HEQ. Interestingly, the Euclidean distance score, $s_{E}$, always delivered better results with images normalised using ZMST whereas the $s_{N}$ and $s_{D}$ scores excelled on the histogram equalised data.

Comparing the best results obtained with each of the matching scores, marked bold in the table, we note that the score based on the proposed gradient metric delivers the best performance overall. In fact the performance achieved with the new method is significantly better than the result obtained with normalised correlation as predicted theo- 


\begin{tabular}{|c|c|c|r|r|r|r|r|}
\hline Score & NOR & \multicolumn{3}{|c|}{ Evaluation set } & \multicolumn{3}{c|}{ Test set } \\
& & FR & FA & TE & FR & FA & TE \\
\hline \hline \multirow{6}{*}{$s_{E}$} & $\times$ & 8.59 & 8.59 & 17.18 & 9.75 & 7.94 & 17.69 \\
& ZM & 9.00 & 9.00 & 18.00 & 9.00 & 8.14 & 17.14 \\
& ZMST & 3.33 & 3.33 & $\mathbf{6 . 6 6}$ & 2.25 & 3.43 & $\mathbf{5 . 6 8}$ \\
& HEQ & 7.83 & 7.83 & 15.66 & 5.50 & 7.35 & 12.85 \\
& FP & 9.17 & 9.17 & 18.34 & 9.25 & 8.31 & 17.56 \\
& FPST & 9.00 & 9.00 & 18.00 & 8.00 & 8.42 & 16.42 \\
& FPHQ & 10.00 & 10.00 & 20.00 & 6.75. & 10.88 & 17.63 \\
\hline \multirow{6}{*}{$s_{N}$} & $\times$ & 3.33 & 3.33 & 6.66 & 3.75 & 3.48 & 7.23 \\
& ZM & 3.33 & 3.33 & 6.66 & 3.50 & 3.49 & 6.99 \\
& ZMST & 3.83 & 3.83 & 7.66 & 3.00 & 3.95 & 6.95 \\
& HEQ & 2.50 & 2.50 & $\mathbf{5 . 0 0}$ & 2.25 & 2.56 & $\mathbf{4 . 8 1}$ \\
& FP & 3.50 & 3.50 & 7.00 & 3.50 & 3.64 & 7.14 \\
& FPST & 3.76 & 3.76 & 7.52 & 3.75 & 3.90 & 7.65 \\
& FPHQ & 3.33 & 3.33 & 6.66 & 2.25 & 3.45 & 5.70 \\
\hline \multirow{6}{*}{$s_{O}$} & $\times$ & 2.95 & 2.95 & 5.90 & 2.50 & 2.80 & 5.30 \\
& HEQ & 1.74 & 1.74 & $\mathbf{3 . 4 8}$ & 1.75 & 1.70 & $\mathbf{3 . 4 5}$ \\
& ZMST & 3.33 & 3.33 & 6.66 & 2.67 & 3.51 & 6.18 \\
\hline
\end{tabular}

Table 1: Performance of the three matching scores on manually registered images

retically. Interestingly, the method gives a very good performance even on the raw data marked by symbol $\times$. This is further improved by photometric normalisation using histogram equalisation. As in the case of the normalised correlation photometric normalisation by removing the image mean and scaling the variance does not have a beneficial effect, in fact the opposite.

The purpose of experimenting with the pseudo registered image data was to establish how robust the approach is to missregistration. As before the training was done using the manually registered images. This is quite realistic even from the operational point of view as it is most likely that client enrollment would be carried out under an operator supervision and any errors in registration could be manually corrected. However, once the training is completed, any future client access claim should be processed automatically. It is therefore pertinent to understand the effect of registration errors on the system performance. Accordingly, the ROC curve was computed using an evaluation set containing images registered with perturbed eye coordinates. The verification system was then tested on an independent test set for which the eye coordinates were also perturbed according to the same error distribution.

The above experiments with the three score measures were limited to raw data, and photometrically normalised images using ZMST and HEQ. The performance degrading effect of missregistration is clearly visible from Table 2. In all cases the best results degrade by a factor of two. The relative ranking in performance is preserved, including the ordering of photometric normalisation methods.

The last set of experiments was conducted using evaluation and test sets with automatically registered images. The results are presented in Table 3. They confirm the general

\footnotetext{
${ }^{5}$ Registration based on manual localised eye coordinates perturbed with 2 standard deviations.
} 


\begin{tabular}{|c|c|c|r|r|r|r|r|}
\hline Score & NOR & \multicolumn{3}{|c|}{ Evaluation set } & \multicolumn{3}{c|}{ Test set } \\
& & FR & FA & TE & FR & FA & TE \\
\hline \hline \multirow{4}{*}{$s_{E}$} & $\times$ & 14.67 & 14.67 & 29.34 & 16.00 & 14.33 & 30.33 \\
& HEQ & 12.75 & 12.75 & 25.50 & 10.00 & 11.80 & 21.80 \\
& ZMST & 4.83 & 4.83 & $\mathbf{9 . 6 6}$ & 4.25 & 4.94 & $\mathbf{9 . 1 9}$ \\
\hline \hline \multirow{4}{*}{$s_{N}$} & $\times$ & 5.33 & 5.35 & 10.68 & 4.50 & 5.53 & 10.03 \\
& HEQ & 4.83 & 4.83 & $\mathbf{9 . 6 6}$ & 4.00 & 4.94 & $\mathbf{8 . 9 4}$ \\
& ZMST & 6.33 & 6.33 & 12.66 & 4.75 & 6.43 & 11.18 \\
\hline \hline \multirow{4}{*}{$s_{O}$} & $\times$ & 4.50 & 4.50 & 9.00 & 4.75 & 4.26 & 9.01 \\
& HEQ & 3.50 & 3.50 & $\mathbf{7 . 0 0}$ & 3.25 & 3.42 & $\mathbf{6 . 7 7}$ \\
& ZMST & 5.00 & 5.00 & 10.00 & 4.25 & 5.11 & 9.36 \\
\hline
\end{tabular}

Table 2: Performance of the three matching scores on pseudo registered images

trends predicted by the experiments on pseudo registered data. In fact the perturbation model used to generate pseudo registered data reflects the statistical distribution of errors of the automatic eye coordinate registration process [4] quite closely. The proposed matching score again achieves the best performance which is more than $20 \%$ better than the next best result. Interestingly, the Euclidean distance measure catches up and overtakes normalised correlation.

\begin{tabular}{|c|c|r|r|r|r|r|r|}
\hline Score & NOR & \multicolumn{3}{|c|}{ Evaluation set } & \multicolumn{3}{|c|}{ Test set } \\
& & FR & FA & TE & FR & FA & TE \\
\hline \hline \multirow{4}{*}{$s_{E}$} & $\times$ & 13.50 & 13.50 & 27.00 & 13.25 & 14.23 & 27.48 \\
& HEQ & 12.33 & 12.39 & 25.69 & 9.25 & 12.86 & 22.11 \\
& ZMST & 4.83 & 4.83 & $\mathbf{9 . 6 6}$ & 4.50 & 4.78 & $\mathbf{9 . 2 8}$ \\
\hline \hline \multirow{4}{*}{$s_{N}$} & $\times$ & 6.00 & 6.00 & 12.00 & 6.25 & 6.20 & 12.45 \\
& HEQ & 4.17 & 4.17 & $\mathbf{8 . 3 4}$ & 5.50 & 4.26 & 9.76 \\
& ZMST & 4.33 & 4.33 & 8.66 & 5.25 & 4.44 & $\mathbf{9 . 6 9}$ \\
\hline \hline \multirow{4}{*}{$s_{O}$} & $\times$ & 4.77 & 4.77 & 9.54 & 5.50 & 4.57 & 10.07 \\
& HEQ & 3.50 & 3.50 & $\mathbf{7 . 0 0}$ & 3.75 & 3.37 & $\mathbf{7 . 1 2}$ \\
& ZMST & 4.67 & 4.67 & 9.34 & 4.25 & 4.57 & 8.82 \\
\hline
\end{tabular}

Table 3: Performance of the three matching scores on automatically registered images

\section{Conclusions}

The performance of face verification systems using a LDA feature space depends on many factors such as image registration, feature space dimension, photometric normalisation, matching score and decision threshold. We investigated the issue of matching score definition in the LDA feature space and established the reason behind the success of the normalised correlation. The understanding gained about the role of metric then naturally led to a novel way of measuring the distance between a probe image and a model, i.e. in the gradient direction of the aposteriori probability of the hypothesised client identity. In extensive experimental studies on a publicly available database ${ }^{1}$ using a standard proto- 
$\mathrm{col}^{2}$ we showed that the proposed metric is consistently superior to both the Euclidean distance and normalised correlation matching scores. The effect of various photometric normalisations ${ }^{3}$ on the matching scores was also investigated.

\section{References}

[1] P. Belhumeur et al, "Eigenfaces vs. Fisherfaces: Recognition Using Class Specific Linear Projection," in Proc. of ECCV'96, pp 45-58, Cambridge, United Kingdom, 1996.

[2] K. Etemad and R.Chellappa, "Face Recognition using Discriminant Eigenvectors," In Proc. of ICASSP'96, pp. 2148-2151, 1996.

[3] K. Jonsson, J. Kittler, Y.Li and G. Matas "Support Vector Machine for Face Authentication," In Proceeding of BMVC'99, pp. 543-553. 1999.

[4] K. Jonsson, G. Matas, and J. Kittler, "Learning Support Vectors for Face Authentication: Sensitivity to Mis-Registrations," In Proceeding of ACCV'00.

[5] Y.P. Li, J. Kittler and G. Matas, "Effective implementation of Linear Discriminant Analysis for face recognition and verification," In Proc. of CAIP'99, pp. 243-242.

[6] C.Liu and H.Wechsler, "Face Recognition Using Evolutionary Pursuit," in Proc. of ECCV'98, Vol. II, pp. 596-612, June 1998.

[7] C.Liu and H.Wechsler, "Enhanced Fisher Linear Discriminant Models for Face Recognition," in Proc. of ICPR'98, pp.1368-1372, 1998.

[8] J.Matas, K.Jonsson and J.Kittler, "Fast face localisation and verification," In Proc. of BMVC'97., pp. 152-161, 1997.

[9] K. Messer, J. Matas, J. Kittler, J. Luettin and G. Maitre, "XM2VTSDB: The Extended M2VTS Database," In Proc. of AVBPA'99, pp.72-77, 1999

[10] B.Moghaddam and A.Pentland, "Probabilistic visual learning for object representation," In S.K. Nayer and T. Poggio,editors, Early Visual learning, pp. 99-130, 1997.

[11] H. Moon and P.J. Phillips, "Analysis of PCA-based Face Recognition Algorithm," In Proc. of FG'98, pp. 205-210, 1998.

[12] S Romdhani, "Face Recognition using Principal Component Analysis," MSc Thesis, Univ. of Glasgow. http://www.elec.gla.ac.uk/ romdhani/pca_doc/pca_doc_toc.htm.

[13] D.L. Swets and J. Weng, "Discriminant Analysis and Eigenspace Partition Tree for Face and Object Reco gnition from Views," In Proc. of FG'96, pp. 192-197, 1996.

[14] M.Turk and A.Pentland, "Eigenface for Recognition," Journal of Cognitive Neuroscience, Vol. 3, no. 1, pp. 70-86, 1991.

[15] W Zhao et al, "Discriminant Analysis of Principal Components for Face Recognition," In Harry Wechsler et al,editors,Face Recognition From theory to Applications, NATO ASI Series, pp. 73-85, Springer, 1998. 